\title{
Tubal abnormalities on hysterosalphingography in primary and secondary infertility in Sokoto, Northwestern-Nigeria
}

\author{
M. Danfulani', G.H. Yunusa', S.M. Ma'aji', S.A. Sa'idu', M.A. Musa² \\ ${ }^{1}$ Department of Radiology, Usmanu Danfodiyo University Teaching Hospital, Sokoto, Nigeria, ${ }^{2}$ Department of Anatomy, College of Health \\ Sciences, Usmanu Danfodiyo University, Sokoto
}

\section{A B S TR A C T}

Background: Hysterosalphingography (HSG) remains a vital method of assessing tubal patency especially in a resource limited setting such as ours and tubal occlusion is the commonest cause of female infertility in the developing world. Objective: To review abnormal tubal pathological findings in hysterosalphingography (HSG) among women being investigated for primary and secondary infertility in Sokoto. Method: This is a retrospective review of HSG results of 317 women being investigated for both primary and secondary infertility. The study was conducted from July 2009 to August 2012. Results: A total of 317 patients were studied. The age ranges of the study participants was from 17-48 years with a mean and standard deviation value of $32.5 \pm 5.5$. Out of the sample size, $139(43.85 \%)$ showed normal HSG findings while 178 (56.15\%) patients had abnormal HSG findings. Tubal abnormalities were found in 112 (35.33\%) of the patients. The commonest tubal abnormality was tubal blockage. Conclusion: The high incidence of tubal pathology revealed in this study showed that HSG is still a very vital tool for infertility work-up. Early detection and prompt treatment of infection in our women should be encouraged as it is the major leading cause of tubal pathologies and consequently this would reduce the contribution of tubal infertility in this environment.

Access this article online Website:

http://nepjol.info/index.php/AJMS

DOI: 10.3126/ajms.v6i2.10581

Key words: Hysterosalphingography, Infertility, Tubal abnormalities

\section{INTRODUCTION}

Hysterosalphingography (HSG) is a radiographic contrast examination that is used to assess the cervical canal, uterine cavity and fallopian tubes, hence is gold standard radiologic examination in assessing anatomic female factor in infertility. ${ }^{1}$ It is performed by cannulating the cervical Os and injecting the contrast medium retrogradely to outline the cervical canal, uterine cavity and both fallopian tubes. It is an invasive procedure but is cheap, rapid and safe diagnostic tool. $^{2}$

Hysterosalphingography can be both diagnostic and therapeutic. ${ }^{3}$ It is indicated in tubal patency assessment, identification of congenital anomalies of genital tract, assessment of uterine cavity, efficacy of tubal sterilization, reversal of tubal surgery and assessment of pathologic secondary amenorhoea. ${ }^{4}$ Its common contra-indication include pelvic inflammatory diseases (PID) and pregnancy. ${ }^{5}$ Severe pain, pelvic infection, haemorrahage and vasovagal attacks constituted some of the common complications of these procedures. ${ }^{5}$

The aim of this study is to document the common tubal abnormalities that are encountered in women who do HSG in this environment; as the tubal factor remain a common aetiological factor in female infertility.

\section{MATERIALS AND METHODS}

This is a retrospective review of 317 HSG investigations of infertile women referred to Nagarta Ultrasound and Radio- 
diagnostic Center-a private radiological center in Sokoto, Northwestern Nigeria. This study was conducted from July 2009 to August 2012. All the patients were referred from different neighboring hospitals and clinics within Sokoto and its environs.

A total of 317 HSG studies of women presented at the center during the course of this study were reviewed. Verbal consent was obtained after fully explaining the procedure to the study participants. The information retrieved from their records included bio data, indication for the examination and the summary of the radiologist reports/findings. Data obtained were entered into Microsoft Excel Spreadsheet and subjected to descriptive statistical analysis using Statistical Package for Social Sciences (SPSS) version 18 for windows.

\section{RESULTS}

A total of 317 patients were involved in the study. Their age ranged from 17-48 years with a mean and standard deviation value $32.5 \pm 5.5$. Figure 1 shows the frequency distribution of the patients according to age ranges. Out of the total sample size, $139(43.85 \%)$ patients showed normal HSG findings while 178 (56.15\%) patients had abnormal HSG findings. Of the 178 patients with abnormalities, $112(35.33 \%)$ had tubal pathologies demonstrated in their HSG. Table 1 shows the pattern of indications for HSG in our study participants.

NB: Almost all patients with tubal abnormality had additional pathology in combination.

Tubal pathology was the commonest findings amongst our patients with 112 (35.33\%) patients out of 178 demonstrated pathologies in their fallopian tubes as shown in Table 2. More than half of the patients with tubal pathology were below the age of 30 years as seen in Figure 2 above. Thereafter tubal pathology findings decreased as the age increases. The commonest tubal pathology in this study include Tubal blockage (unilateral or bilateral), Hydrosalphinges (Unilateral or bilateral), tubal spasms, perifimbrial adhesions.

\section{DISCUSSIONS}

HSG is highly sensitive in detecting Uterine and tubal abnormalities and this makes it an important diagnostic tool for uterine and tubal condition. Radiologic studies are valuable non-operative procedures for identifying tubal and uterine pathology although laparoscopy and dye test are more informative. ${ }^{6,7}$ The most suitable period to perform HSG is towards the end of the first week (ten days rule) after menstrual period when the isthmus of the fallopian tubes is most distensible easing demonstration of tubes by filled contrast medium. ${ }^{4}$ It is however avoided in the second half of the cycle because of fear of irradiating an ongoing pregnancy and because the thickened secretory endometrium increases the risk of venous intravasation which makes proper assessment of the fallopian tubes difficult. 8,9

The Figure 3 above shows a normal HSG examination of one of our study participant while Figure 4 and 5 shows HSG images of some patients with tubal abnormalities.

In this study it is discovered that the incidence of Secondary infertility was higher than the incidence of primary infertility this is similar to findings of others in the sub-region, ${ }^{5,9-11}$ However it differs from reports from some other studies where primary infertility was most common. ${ }^{12-14}$

Tubal pathology was the largest abnormality observed in our study; of the 317 patients reviewed $112(35.33 \%)$ showed findings in the fallopian tubes: Right tubal block was more preponderant over the left tubal block, this

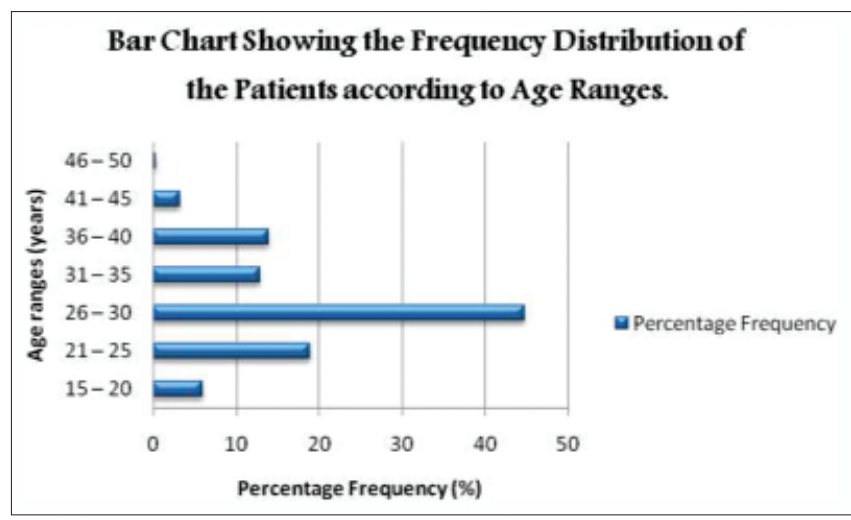

Figure 1: Bar chart showing the frequency distribution of the patients according to age ranges

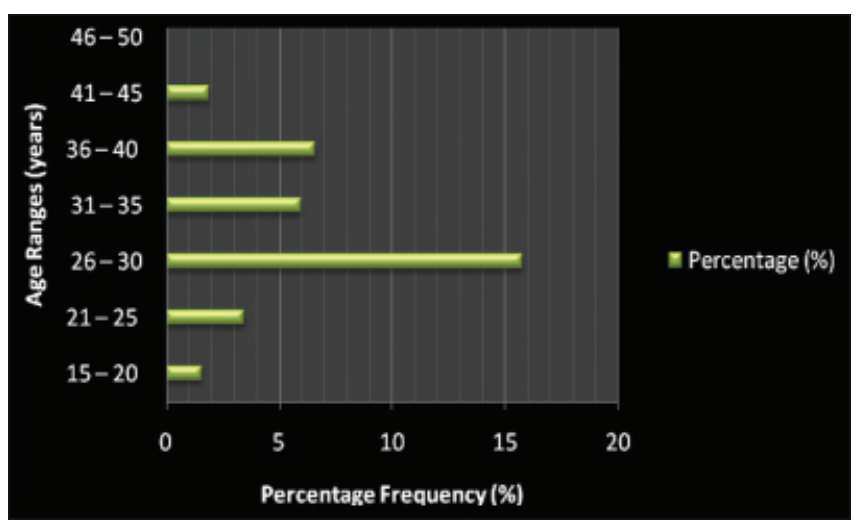

Figure 2: Bar chart showing the distribution of tubal pathology according to age ranges. 


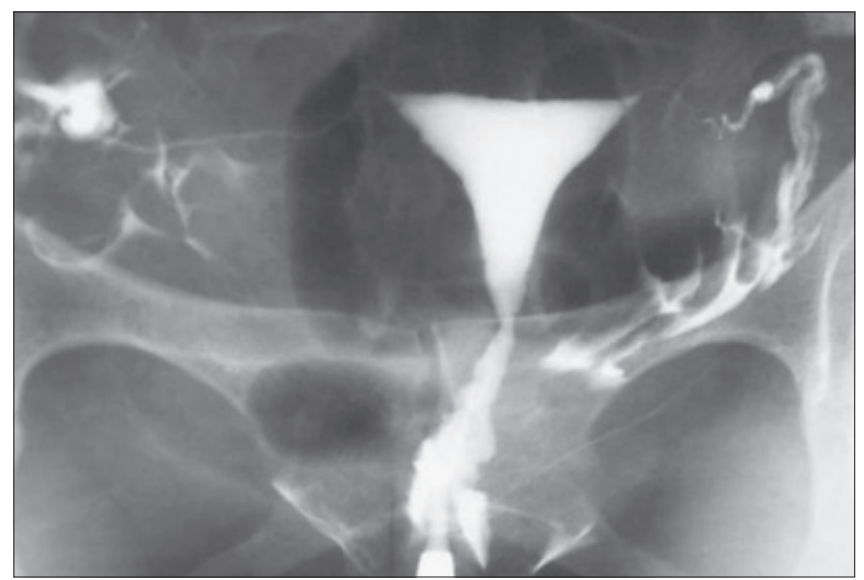

Figure 3: Showing a typical normal HSG image

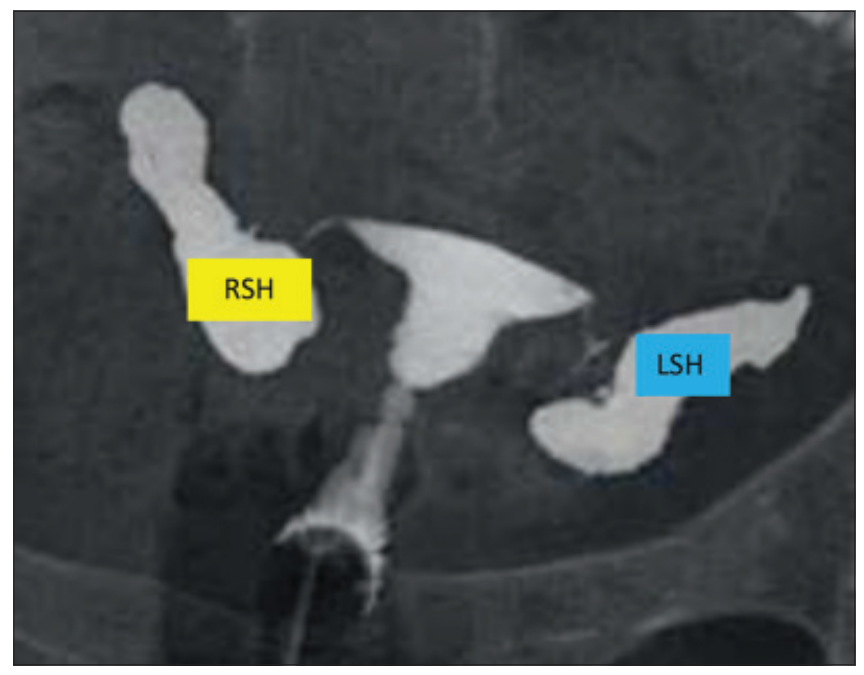

Figure 4: Showing HSG image of a patient with Bilateral Hydrosalpinges. RSH: Right sided hydrosalphinx and LSH: Left sided hydrosalphinx

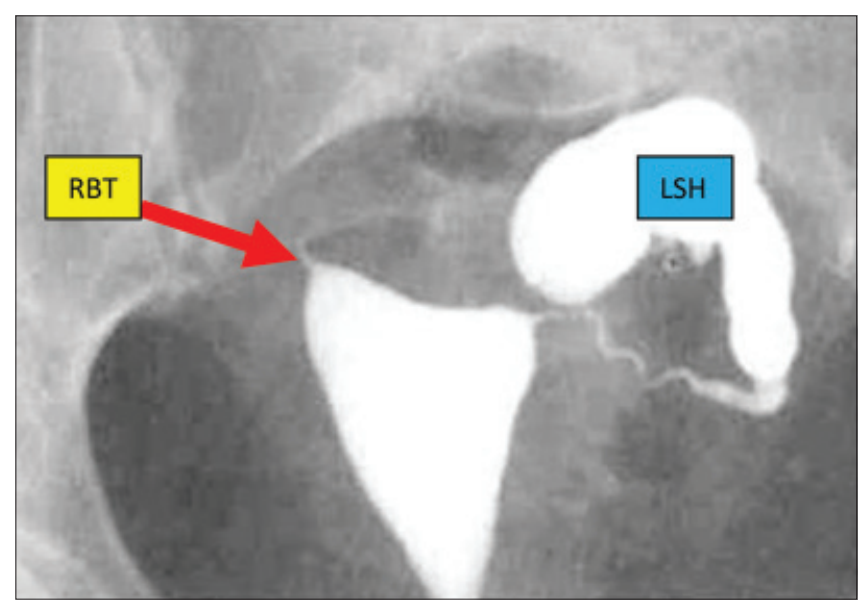

Figure 5: Showing HSG image of a patient with left sided hydrosalphinx (LSH) and right blocked tube (RBT)

findings agrees with what was reported by Adetiloye, ${ }^{15}$ but is in contrast to findings elsewhere where bilateral tubal block constituted the largest. ${ }^{16,17}$

\begin{tabular}{|c|c|c|}
\hline \multirow{2}{*}{$\begin{array}{l}\text { Age in } \\
\text { years }\end{array}$} & \multicolumn{2}{|c|}{ Indication for HSG } \\
\hline & $\begin{array}{c}\text { Primary infertility } \\
N=143\end{array}$ & $\begin{array}{c}\text { Secondary infertility } \\
\qquad N=174\end{array}$ \\
\hline $15-20$ & 14 & 5 \\
\hline $21-25$ & 37 & 23 \\
\hline $26-30$ & 49 & 93 \\
\hline $31-35$ & 18 & 23 \\
\hline $36-40$ & 19 & 25 \\
\hline $41-45$ & 6 & 4 \\
\hline $46-50$ & 0 & 1 \\
\hline
\end{tabular}

\section{Table 2: Showing pattern of tubal pathology findings amongst our HSG patients}

\begin{tabular}{lcc} 
Findings & Number & Percentage \\
\hline Normal & 139 & 41.7 \\
Uterine+cervical findings & 130 & 39 \\
Fallopian tubes & & \\
$\quad$ Rt tubal block & 19 & 3.7 \\
Lt tubal block & 10 & 45 \\
$\quad$ Bilateral tubal & 15 & 1.8 \\
Hydrosalphinges & & 3 \\
$\quad$ Rt sided hydrosalphinx & 6 & 3.6 \\
Lt sided hydrosalphinx & 10 & 0.3 \\
$\quad$ Bilateral hydrosalphinx & 12 & 12 \\
Pelvic adhesions & 1 & \\
Tubal pathology co-existing & 40 & \\
with other findings & & \\
\hline NB: Total patients with tubal pathology=112 & &
\end{tabular}

Infection is the commonest single most important cause of infertility in our environment as it is demonstrated by the high incidence of tubal pathologies as seen in this and many previous studies. ${ }^{16-18}$ Consequently primary prevention and prompt treatment of this infection is of paramount importance and should be encourage in the reduction of high incidence of tubal infertility in our environment.

Hydrosalphinges was the next common tubal pathology noted in this study; Bilateral hydrosalphinges was more common this is similar to findings in study in Port Harcourt where the incidence was $(5 \%) .{ }^{19}$ It was followed by left sided Hydrosalphinx which occurred in 10 (3\%) patients.

In conclusion the high incidence of tubal pathology reveal by this study shows that HSG is still a very vital tool for infertility work-up. Early detection and prompt treatment of infection in our women should be encouraged as it is the major leading cause of tubal pathologies and consequently this would reduce the contribution of tubal infertility in this environment.

\section{REFERENCES}

1. Oguntoyinbo $\mathrm{AE}$, Amole $\mathrm{AO}$ and Komolafe OF. Sonographic Assessment of tubal patency in the investigation of female 
infertility in Ilorin, Nigeria. Afrij Reproductive Health 2001; 5: 100-105.

2. Siegler AM. Hysterosalphingography, Fertility Steril 1983; 40: 139-159.

3. SchwabeMG, ShapiroSSandHaningRV. Hysterosalphingography with oil contrast medium enhances fertility in patients with infertility of unknown etiology; fertility and sterility. 1983; 40: 604606.

4. Ronald GG. The female reproductive systems in: Grainger RG, Allison DJ, editors. Diagnostic Radiology. Vol. 3, Philadelphia: Churchill Livingstone; 1993; 1809-1869.

5. Kaishima JA, Dani PAand Ekedigwe JE. Hysterosalphingographic evaluation of 998 consecutive infertile women in Jos, Nigeria. Int.J Gynaecol Obst., 2010; 108 (3): 255-257.

6. Ladipo OA. An evaluation of 576 hysterosalphingograms on infertile women. Infertility 1979; 2: 63-70.

7. Idrisa A, Ojiyi E and Hamidu AU. Hysterosalphingography versus laparoscopy in the evaluation of female infertility in Maiduguri, Nigeria. Tropical J Obstet Gynaecol 2003; 20: 20-23.

8. Crofton M. gynecological imaging. Textbook of Radiology and Imaging, $7^{\text {th }}$ ed, vol 2. 2007. P. 1069.

9. Bukar M, Mustapha Z, Takai UI and Tahir A. Hysterosalphingographic findings in infertile women: A seven year review. Niger J clin pract. 2011; 14: 168-170.

10. Okafor $\mathrm{CO}$, Okafor $\mathrm{Cl}$, Okpala $\mathrm{OC}$ and Umeh E. The pattern of hysterosalphingographic findings in women being investigated for infertility in Nnewi, Nigeria. Niger $\mathrm{J}$ clin pract. 2010; 13 (3): 264-267.

11. Ibekwe PC, Udensi $A M$ and Imo AO. Heterosalphingographic findings in patients with infertility in south eastern Nigeria. Niger J Med. 2010; 19 (2): 165-167.

12. Bello TO. Pattern of tubal pathology in infertile women on hysterosalphingography in Illorin, Nigeria. Annals of African Medicine 2004: 3 (2): 77-79.

13. Cisse R, Houge C, Quadraogo A, Thieba B, Tap soba T, Quedraogo CM, et al. Features of hysterosalphingography performed in Burkina Faso. J Radiology. 2003. 83 (3): 361-364.

14. Sinawat $\mathrm{S}$, Pattamadilok $\mathrm{J}$ and Seejorn $\mathrm{K}$. Tubal abnormalities in Thai infertile female. Journal of Medical Association of Thailand. 2005; 88 (6): 723-727.

15. Adetiloye VA. Hysterosalphingography in the investigation of infertility: experience with 248 patients. West Afr J med 1993; 12: 191-196.

16. Mgbor SO. Pattern of hysterosalphingographic findings in gynaecological patients in Enugu. Nig Med J. 2006; 47: 14-16.

17. Adinma JB, Adinma $E$ and Okpala OC. Hysterosalphingography in management of infertility. Bilatera tubal occlusion. West Afr J Radiol. 1995; 3: 29-33.

18. Marinho AO. Aetiological factors in infertility: a review of 277 Nigerian Infertile Couples. West Afr J med 1986; 5: 69-73.

19. Nwanko NC and Akani Cl. Pattern of findings in infertility in port Harcourt. West Afr J Radiol 2005; 12 (1): 15-19.

DM - Concept and Study Design, Analysis of Data, Interpretation and Manuscript preparation; SSA - Study Design and Manuscript preparation; YGH \& MSM - Study Design and Critical revision of manuscript; MMA - Analysis of Data, Literature Search and Manuscript preparation. 